\title{
Income-related inequalities in preventive and curative dental care use among working-age Japanese adults in urban areas: a cross-sectional study
}

Keiko Murakami ${ }^{1} 2^{*}$, Jun Aida ${ }^{3}$, Takayoshi Ohkubo ${ }^{1}$ and Hideki Hashimoto ${ }^{2}$

\begin{abstract}
Background: Preventive dental care use remains relatively low in Japan, especially among working-age adults. Universal health insurance in Japan covers curative dental care with an out-of-pocket payment limit, though its coverage of preventive dental care is limited. The aim of this study was to test the hypothesis that income inequality in dental care use is found in preventive, but not curative dental care among working-age Japanese adults.

Methods: A cross-sectional survey was conducted using a computer-assisted, self-administered format for community residents aged 25-50 years. In all, 4357 residents agreed to participate and complete the questionnaire (valid response rate: $31.3 \%$ ). Preventive dental care use was measured according to whether the participant had visited a dentist or a dental hygienist during the past year for dental scaling or fluoride or orthodontic treatments. Curative dental care use was assessed by dental visits for other reasons. The main explanatory variable was equivalent household income. Logistic regression analyses with linear trend tests were conducted to determine whether there were significant income-related gradients with curative or preventive dental care use.

Results: Among the respondents, $40.0 \%$ of men and $41.5 \%$ of women had used curative dental care in the past year; $24.1 \%$ of men and $34.1 \%$ of women had used preventive care. We found no significant income-related gradients of curative dental care among either men or women ( $p=0.234$ and $p=0.270$, respectively). Significant income-related gradients of preventive care were observed among both men and women $(p<0.001$ and $p=0.003$, respectively). Among women, however, income-related differences were no longer significant $(p=0.126)$ after adjusting for education and other covariates. Compared with men with the lowest income, the highest-income group had a 1.79-fold significantly higher probability for using preventive dental care.

Conclusions: The prevalence of preventive dental care use was lower than that of curative care. The results showed income-related inequality in preventive dental care use among men, though there were no significant income-related gradients of curative dental care use among either men or women. Educational attainment had a positive association with preventive dental care use only among women.
\end{abstract}

Keywords: Dental care, Dental insurance, Income, Inequality, Japan

\footnotetext{
* Correspondence: mkeiko-tky@umin.ac.jp

'Department of Hygiene and Public Health, School of Medicine, Teikyo

University, 2-11-1 Kaga, Itabashi-ku, Tokyo 173-8605, Japan

2Department of Health and Social Behavior, School of Public Health, The

University of Tokyo, 7-3-1 Hongo, Bunkyo-ku, Tokyo 113-0033, Japan

Full list of author information is available at the end of the article
} 


\section{Background}

Many studies have consistently shown that an incomerelated inequality exists in dental care use [1-6]. Affluent people have better access to dental care than the less affluent despite the fact that dental care needs are more severe among the less affluent [7-9]. The magnitudes of inequalities were larger than those for other types of health care, which is often attributed to the fact that dental insurance is not provided publicly and has to be paid for either out-of-pocket or through private insurance coverage in many developed countries. Japan is one of the exceptions. Since the start of its universal health insurance coverage in 1961, the system has covered inpatient, outpatient, and dental care [10]. Unlike medical care, however, dental care allows extra charges out of insurance coverage, and it covers preventive dental care only for limited conditions [11].

There are extensive prevention possibilities, and prevention can actually save resources especially in case of dental care [12]. Dental care use in Japan has traditionally been treatment-oriented, and the prevalence of preventive dental care use remains relatively low especially among working-age adults; for example, the percentages of those who reported having dental check-ups in the past year were $29.4 \%$ of people in their twenties, $32.2 \%$ of those in their thirties or forties, while that was $41.4 \%$ of those in their sixties [13]. The concept of prevention has become widespread in recent decades, and there has been improvement in oral health behaviors including in Japan $[14,15]$. However, such spreading of preventive behaviors is often accompanied by inequalities across socioeconomic positions, due to differential uptake of new information and skills. In other words, increased preventive behavior often results in more disadvantage among lower social groups than higher ones [16]. In addition to individual education for preventive behavior, a system to facilitate access to preventive care is imperative to drive oral health improvement on a population level. In this regards, public insurance coverage for preventive care would narrow the gap.

As mentioned above, some forms of preventive dental care are not covered by insurance in Japan, so we used the situation to test a hypothesis that the income gap in service use is found in preventive dental care but not in curative dental care, which is covered by insurance. There might be significant value to examine how the different coverage between curative and preventive care would affect income-related inequalities in dental care use in one country.

\section{Methods \\ Data}

The data were derived from the Japanese Study of Stratification, Health, Income, and Neighborhood (J-SHINE). The
J-SHINE survey was conducted between October 2010 and February 2011 in four municipalities in and around the Tokyo metropolitan area. The survey participants were community residents aged $25-50$ years who were randomly selected from the residential registry. The sampling strategy for this survey is described in detail elsewhere [17]. Of 13920 residents who were randomly selected, after excluding those individuals who had moved, were absent, or could not be located, survey staffs could have contact with 8408 residents (contact success rate: 60.4\%). Those individuals who agreed to complete the survey were asked to complete the questionnaire in a computerassisted, self-administered format unless the participants requested a face-to-face interview. Among them, a total of 4357 residents agreed to participate and complete the questionnaire (response rate: $31.3 \%$; cooperation rate: $51.8 \%$ ). From the responses received, we used an analytic sample of 3083 individuals who did not have missing values for household income, age, gender, self-assessed oral health, marital status, educational attainment, work status, and dental care use $(22.1 \%$ of the originally selected sample). Because this attrition was mainly due to nonresponse on income, we applied a multiple imputation of income and obtained similar results. We therefore present only the results without imputation of income. The secondary use of the data was approved by the 2011 data management committee of the J-SHINE research group, with personally identifiable information deleted to ensure confidentiality.

\section{Measures}

Curative dental care use in the past year was measured through self-reporting in response to the question, "In the past year, have you been seen by a dentist or a dental hygienist? Exclude use for dental scaling and fluoride and orthodontic treatments." Preventive dental care use in the past year was measured through self-report responses to the question, "In the past year, have you been seen by a dentist or a dental hygienist for dental scaling or fluoride or orthodontic treatments?"

Each respondent was asked to select his/her total annual household income from among 15 categories: <250, 250-500, 500-750, 750-1000, 1000-1250, 1250-1500, 1500-2000, 2000-3000, 3000-4000, 40005000, 5000-7500, 7500-10000, 10000-15000, 1500020000 , and $>20000$ thousand Japanese yen. It was made clear that household income included stock dividends, extra income, and additional income. The equivalent household incomes were derived from adjustment for household size using the OECD-modified equivalence scale [18].

The respondents were sorted into three categories of educational attainment: high school or less (elementary, junior high, or senior high school), two-year college or 
special training school, and university or higher (university or graduate school). Self-assessed oral health was determined by answers to the question, "How would you describe the health of your teeth and gums? Would you say it is excellent, very good, good, fair, or poor?" $[19,20]$. Since the number of respondents in the "poor" stratum category was small, "poor" and "fair" categories were combined into one "poor/fair" category for analysis purposes.

\section{Statistical analysis}

Differences in the proportions of selected characteristics of respondents by gender were assessed by chi-square test. Multiple logistic regression analyses were conducted to examine the association of household income with curative and preventive dental care use. Crude odds ratios, and odds ratios adjusted for other social and dental variables (age, marital status, educational attainment, work status, city of residence, and self-assessed oral health), and their 95\% confidence intervals, were calculated. A trend test was also conducted to test the linear trend between household income and dental care use. Some previous studies found gender differences in the association between socioeconomic status and health outcomes. For example, one study on elderly Japanese people identified gender differences among the factors related to dental care access, including income indicators [21]. To test the significance of distinct associations between socioeconomic status (i.e., income and educational attainment) and dental care use according to gender, the interaction term between each socioeconomic indicator and gender was entered into the model. The results showed no significant interaction between socioeconomic status and gender for curative dental care use (income $p=0.816$ and educational attainment $p=0.397$, respectively). For preventive dental care, the interaction term between educational attainment and gender was statistically significant $(p=0.022)$, but no significant interaction between income and gender was found $(p=0.500)$. On the basis of these results, we present only the results of the analyses conducted separately for men and women.

Statistical analysis was conducted using STATA (release 12; Windows version). All analyses were two-tailed, and $p$-values less than 0.05 were considered statistically significant.

\section{Results}

Table 1 presents the sociodemographic characteristics of the respondents by gender. Men were more likely to be highly educated, be currently working, have higher household income, and were less likely to be married by formal or common law than women.

Table 2 shows prevalence, the odds ratios (ORs) and 95\% confidence intervals (CIs) for curative dental care use by gender. The percentages of those who used curative dental care during the past year were $40.0 \%$ and $41.5 \%$ among men and women, respectively. No significant income-related gradients of curative care were found among either men or women (trend test $p=0.234$ and $p=0.270$, respectively). In addition, none of age, marital status, educational attainment, work status, or city of residence was significantly associated with curative care. The likelihood of curative dental care use was higher among those who assessed their own oral health as worse than among those as better.

Table 3 presents prevalence, the ORs and 95\% CIs for preventive dental care use by gender. More women than men had used preventive dental care during the past year (34.1\% compared with $24.1 \%$ ). Among men, there was a significant income-related gradient of preventive care use, even after adjusting for covariates (trend test $p=0.001$ ). Compared to men with the lowest income, only those in the highest income group had significantly higher probability of using preventive dental care $(p=0.003)$. Women in higher household income groups were also more likely to use preventive dental care (trend test $p=0.003$ ). However, after adjusting for education and other covariates, the income-related difference in preventive care substantially decreased and was no longer significant (trend test $p=0.126$ ). We found a significant association between educational attainment and preventive care among women, although this was not seen for men. The likelihood of preventive dental care use was higher among those who assessed their own oral health as better than among those as worse.

\section{Discussion}

This study explored income-related inequality in curative and preventive dental care use among working-age Japanese men and women. The prevalence of preventive dental care use was lower than that of curative one. We found no significant income-related gradients of curative dental care among either men or women. Significant income-related gradients in preventive dental care were observed for both men and women. Among women, however, the income-related difference was no longer significant after adjusting for education and other covariates. Educational attainment was positively associated with preventive dental care use only for women.

The present study found income-related inequalities in preventive dental care use among men, but no significant inequalities in curative one, as expected from the insurance coverage status of the two types of dental care. It is noteworthy that use of curative dental care, which is mostly covered by public insurance in Japan, showed no income-related inequality. In Canada, the income-related inequality in frequent preventive dental care was larger than that measured in total dental care [22]. In European countries, analyses of the data from a representative sample 
Table 1 Characteristics of respondents by gender: Japanese Study of Stratification, Health, Income, and Neighborhood (J-SHINE)

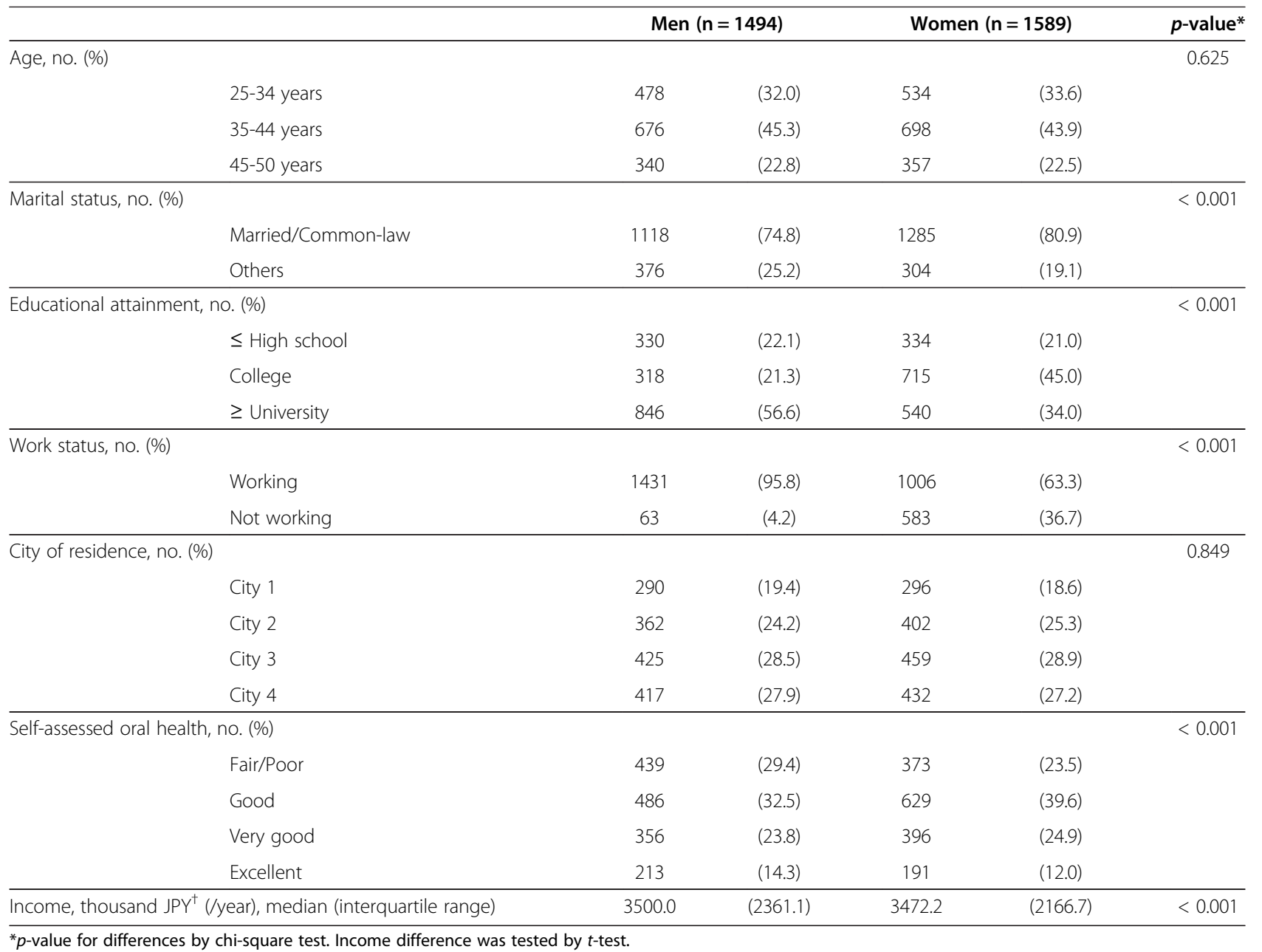

${ }^{*} p$-value for differences by chi-square test. Income difference was tested by $t$-test

†JPY: Japanese Yen.

aged 50 and over have also shown that a huge proportion of income-related inequalities in dental care use was attributable to inequalities in preventive care, but not to differential use of operative care alone [23]. The present findings are generally in agreement with these previous studies.

The results of gender differences in income-related inequalities are probably derived from the argument that income may not reflect a woman's position in the social hierarchy [24]. Several studies have demonstrated that social inequalities in health are greater when assessing the most dominant social position in the household than a woman's own social position and that this applies in Japan [25]. A previous study using a representative sample in Japan showed that household income may exert a direct effect on psychological distress among men, but not among women [26].

One study on elderly Japanese people found that there was a tendency only for men with low equivalent income not to visit dental clinics regularly [21]. In light of these results, factors other than income may affect preventive dental care among women. Women tend to use preventive health care services more frequently than men, partly because women report more interest in health [27]. Dental scaling, which accounts for a considerable proportion of preventive dental care, is of mainly two types: one is aftercare treatment for periodontal disease; the other is purely preventive care, irrespective of periodontal disease. The present study showed that the likelihood of preventive dental care use was higher among women than among men, which implies that women tend to use preventive dental care irrespective of periodontal disease. No gender difference in the likelihood of using curative dental care was observed. Such preventive care may be associated with knowledge of or consciousness related to dental health $[12,15]$; therefore, our results indicate that educational attainment was associated with preventive care only among women. 
Table 2 Prevalence, odds ratio (OR) and 95\% confidence interval (CI) for curative dental care use

\begin{tabular}{|c|c|c|c|c|c|c|c|c|c|c|}
\hline \multirow{4}{*}{$\begin{array}{l}\text { Total } \\
\text { Income quartiles }\end{array}$} & \multirow{4}{*}{$\begin{array}{c}\% \\
40.0 \\
\end{array}$} & \multicolumn{4}{|c|}{ Men $(n=1494)$} & \multirow{4}{*}{$\begin{array}{c}\% \\
41.5 \\
\end{array}$} & \multicolumn{4}{|c|}{ Women $(n=1589)$} \\
\hline & & \multicolumn{2}{|c|}{ Crude OR $(95 \% \mathrm{Cl})$} & \multicolumn{2}{|c|}{ Adjusted $\mathrm{OR}^{*}(95 \% \mathrm{Cl})$} & & \multicolumn{2}{|c|}{ Crude OR (95\% Cl) } & \multicolumn{2}{|c|}{ Adjusted OR* $(95 \% \mathrm{Cl}$} \\
\hline & & & & & & & & & & \\
\hline & & & $=0.440$ & & 0.234 & & & $=0.332$ & & 0.270 \\
\hline 1 (lowest) & 37.5 & 1.00 & & 1.00 & & 42.9 & 1.00 & & 1.00 & \\
\hline 2 & 42.1 & 1.21 & $(0.91-1.61)$ & 1.29 & $(0.96-1.74)$ & 35.8 & 0.74 & $(0.57-0.98)$ & 0.75 & $(0.57-0.99)$ \\
\hline 3 & 38.9 & 1.06 & $(0.79-1.43)$ & 1.12 & $(0.82-1.54)$ & 46.2 & 1.14 & $(0.85-1.53)$ & 1.17 & $(0.86-1.58)$ \\
\hline 4 (highest) & 41.4 & 1.18 & $(0.88-1.57)$ & 1.28 & $(0.94-1.75)$ & 43.0 & 1.00 & $(0.76-1.33)$ & 1.03 & $(0.76-1.40)$ \\
\hline \multicolumn{11}{|l|}{ Age } \\
\hline 25-34 years & 41.6 & 1.00 & & 1.00 & & 39.3 & 1.00 & & 1.00 & \\
\hline $35-44$ years & 35.8 & 0.78 & $(0.61-0.99)$ & 0.72 & $(0.56-0.93)$ & 41.8 & 1.11 & $(0.88-1.40)$ & 1.04 & $(0.82-1.32)$ \\
\hline $45-50$ years & 45.9 & 1.19 & $(0.90-1.57)$ & 1.05 & $(0.78-1.42)$ & 44.3 & 1.22 & $(0.93-1.61)$ & 1.08 & $(0.81-1.43)$ \\
\hline \multicolumn{11}{|l|}{ Marital status } \\
\hline Married/Common-law & 41.1 & 1.00 & & 1.00 & & 42.3 & 1.00 & & 1.00 & \\
\hline Others & 36.7 & 0.83 & $(0.65-1.06)$ & 0.84 & $(0.65-1.09)$ & 38.2 & 0.84 & $(0.65-1.09)$ & 0.81 & $(0.61-1.07)$ \\
\hline \multicolumn{11}{|l|}{ Educational attainment } \\
\hline$\leq$ High school & 40.3 & 1.00 & & 1.00 & & 42.8 & 1.00 & & 1.00 & \\
\hline College & 40.3 & 1.00 & $(0.73-1.37)$ & 1.04 & $(0.75-1.43)$ & 42.4 & 0.98 & $(0.76-1.28)$ & 1.01 & $(0.77-1.32)$ \\
\hline$\geq$ University & 39.7 & 0.98 & $(0.75-1.26)$ & 1.06 & $(0.80-1.39)$ & 39.6 & 0.88 & $(0.66-1.16)$ & 0.91 & $(0.67-1.22)$ \\
\hline \multicolumn{11}{|l|}{ Work status } \\
\hline Working & 40.2 & 1.00 & & 1.00 & & 42.9 & 1.00 & & 1.00 & \\
\hline Not working & 34.9 & 0.80 & $(0.47-1.36)$ & 0.90 & $(0.52-1.58)$ & 39.1 & 0.85 & $(0.69-1.05)$ & 0.85 & $(0.68-1.06)$ \\
\hline \multicolumn{11}{|l|}{ City of residence } \\
\hline City 1 & 42.4 & 1.00 & & 1.00 & & 45.6 & 1.00 & & 1.00 & \\
\hline City 2 & 38.4 & 0.85 & $(0.62-1.16)$ & 0.86 & $(0.62-1.19)$ & 42.8 & 0.89 & $(0.66-1.21)$ & 0.92 & $(0.67-1.25)$ \\
\hline City 3 & 40.2 & 0.91 & $(0.68-1.24)$ & 0.92 & $(0.67-1.25)$ & 42.3 & 0.87 & $(0.65-1.17)$ & 0.92 & $(0.68-1.25)$ \\
\hline City 4 & 39.3 & 0.88 & $(0.65-1.19)$ & 0.89 & $(0.65-1.21)$ & 36.8 & 0.69 & $(0.51-0.94)$ & 0.74 & $(0.54-1.00)$ \\
\hline \multicolumn{11}{|l|}{ Self-assessed oral health } \\
\hline Fair/Poor & 50.1 & 1.00 & & 1.00 & & 48.3 & 1.00 & & 1.00 & \\
\hline Good & 37.2 & 0.59 & $(0.45-0.77)$ & 0.59 & $(0.45-0.77)$ & 42.1 & 0.78 & $(0.60-1.01)$ & 0.79 & $(0.61-1.02)$ \\
\hline Very good & 37.1 & 0.59 & $(0.44-0.78)$ & 0.57 & $(0.43-0.77)$ & 38.1 & 0.66 & $(0.50-0.88)$ & 0.69 & $(0.52-0.93)$ \\
\hline Excellent & 30.1 & 0.43 & $(0.30-0.61)$ & 0.42 & $(0.29-0.59)$ & 33.5 & 0.54 & $(0.38-0.78)$ & 0.56 & $(0.39-0.81)$ \\
\hline
\end{tabular}

${ }^{*}$ Adjusted for all other variables shown in the table.

${ }^{\dagger} p$-value of trend test.

Because the data were cross-sectional in nature, the survey assessed dental care use in the past year, whereas oral health was assessed at the time of the survey only. There were therefore temporal inconsistencies between actual dental care use and oral health status. Previous studies have shown that self-assessed oral health was positively associated with dental care use; this result confirmed that self-assessed oral health status probably represents the outcome of dental care rather than the need for it $[22,28]$. In the present study, self-assessed oral health showed opposite gradients for the two types of dental care: those who assessed their own oral health as better were less likely to use curative, but more likely to use preventive dental care than others, which was similar to the results of a previous study in the United States [29]. The result of preventive care is likely due to the reverse causality.

These findings have some implications for dental care policy. Universal coverage seems to function effectively for curative dental care, while further steps to promote preventive dental care should be needed, particularly considering the impact of income on its use. One promising strategy which does not rely on individual choice may be to include oral health as part of the mandatory annual general health check in Japan. The Japanese health care system has the unique characteristic of including a 
Table 3 Prevalence, odds ratio (OR) and $95 \%$ confidence interval $(\mathrm{CI})$ for preventive dental care use

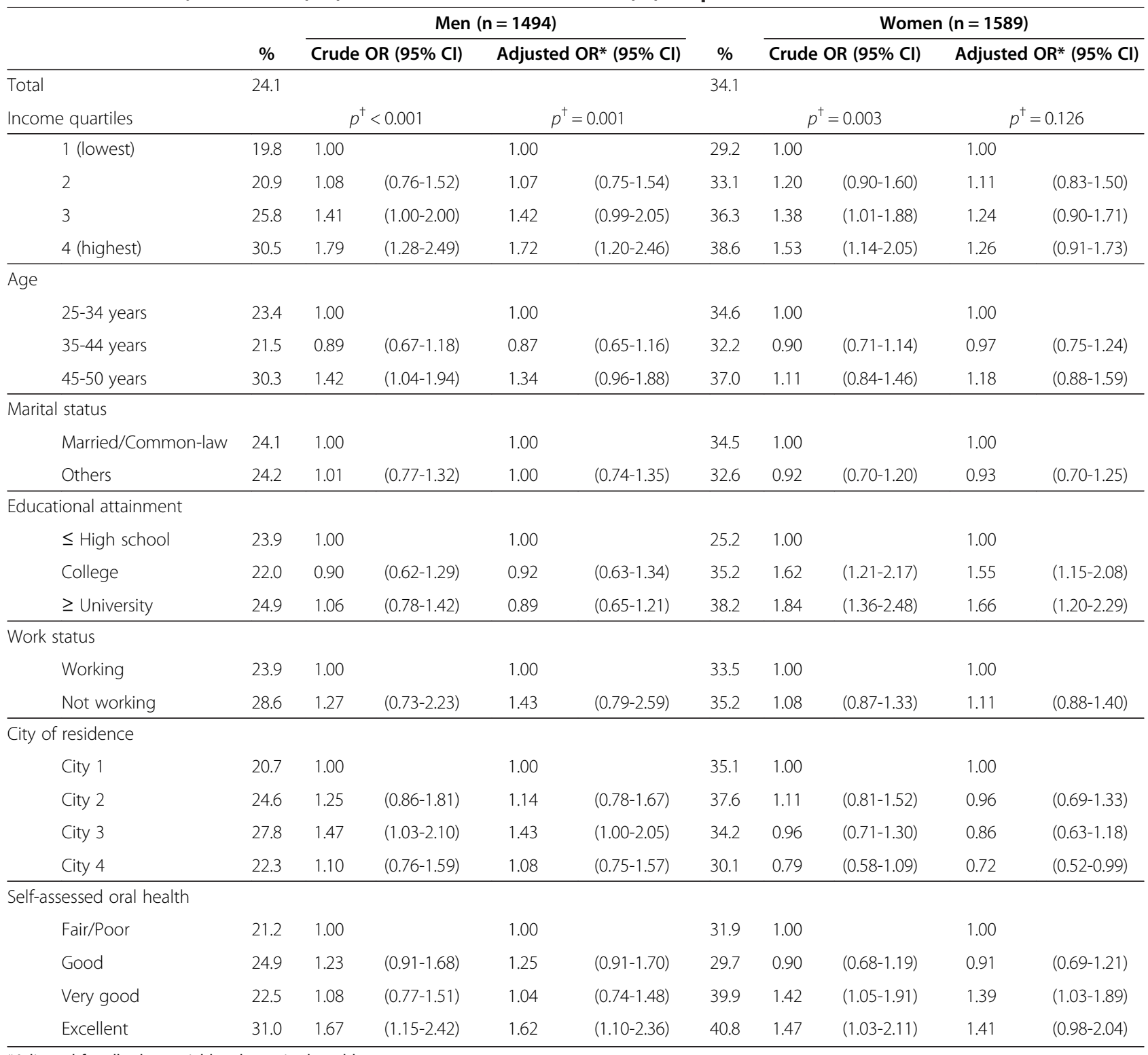

*Adjusted for all other variables shown in the table.

${ }^{\dagger} p$-value of trend test.

mandatory annual health checkup for all, but a dental checkup is not included in most settings. In a randomized controlled trial in the UK, the inclusion of a dental checklist within the preventive health checkup for elderly patients together with help arranging a dental appointment has shown that the offer of dental care was taken up most readily by those with current oral health problems, pain, and no regular dentist [30]. Although economic evaluation in the field of dentistry is undeniably lacking [31], the introduction and cost-effectiveness of these promising strategies may be important.

Some limitations of the present study should be considered. First, the response rate was low. This was largely due to the low contact success rate (60.4\%). Survey staffs attempted to contact potential participants at least six times, but over one-third of them could not be reached. Several previous studies have examined nonparticipation in surveys by linking it with register information, and found that compared with respondents, non-respondents have low socioeconomic status such as income and educational attainment $[32,33]$. If such a non-response bias existed in the present study, socioeconomic inequalities in dental care use would be underestimated. However, the sample obtained was fairly comparable with the target population in terms of age and sex distribution, and percentages of graduates of high school or less in Census 
2010 [17]. Second, the sampled municipalities were all located in urban areas, where the demographic structure and access to dental care may be different from rural areas. The findings should therefore be generalized only with caution. However, a previous study revealed that there is an oversupply of dentists in Japan, which has reached a level such that the nationwide distribution of dentists has become relatively homogenous [34]. Third, the measurement of dental care use remains controversial. The present study examined curative and preventive dental care separately, which is its strength. However, it could be difficult to distinguish use for exclusively preventive purposes from that for both curative and preventive purposes. Unfortunately, the definition of dental care (both preventive and curative) differs among studies or countries [22,29]. To our knowledge, previous studies have not defined or examined preventive dental care in Japan in detail. Because dental scaling, fluoride, and orthodontic treatments are a better proxy for placing a high priority on good oral health than other dental care (such as fillings, crowns, and root canals), we believe that the definition of preventive dental care in the present study is suitable at the moment. In addition, the quality of dental care used could not be examined because of data limitations. Finally, the J-SHINE data were based on self-reports. Income data could be subject to bias due to under-reporting, over-reporting, and a nonnegligible number of missing values. More sophisticated methods for eliciting accurate income information (e.g., in-person interviews) have been developed, but of course these come at a cost of having to devote more space and time to collect these data [35]. In addition, even these methods could not eliminate reporting biases completely. Also, income data in this study was divided into discrete numbers of categories, which could lead to measurement error. In general, people in lower income groups are less likely to report their income status, which could lead to bias. Compared with nationally representative data, such as the Comprehensive Survey of the Living Conditions of People on Health and Welfare in Japan, 2010 [36], the mean equivalent income in the present study was higher than national per capita income. This result might have led to underestimation of the association. However, as mentioned above, our analysis using a multiple imputation of income showed similar results, making it less likely that non-response on income substantially affected the results.

\section{Conclusions}

Among working-age Japanese men and women in urban areas, the prevalence of preventive dental care use was lower than that of curative care. The results showed income-related inequality in preventive dental care use among men, though there were no significant incomerelated gradients of curative dental care use among either men or women. In addition, educational attainment had a positive association with preventive dental care use only among women.

\section{Competing interests}

The authors declare that they have no competing interests.

\section{Authors' contributions}

KM conceived the study, performed the statistical analysis, and drafted the manuscript as the principal author. JA contributed to the study design, data interpretation, and writing the manuscript. TO contributed to data interpretation and writing the manuscript. HH was the principal investigator, participated in designing study, and helped to write the manuscript as the supervisor of KM. All authors read and approved the final manuscript.

\section{Acknowledgements}

This research was supported by a Grant-in-Aid for Scientific Research on Innovative Areas (No. 21119002) from the Ministry of Education, Culture, Sports, Science and Technology, Japan. The authors would like to thank Dr. Yasuki Kobayashi for his helpful comments on the early draft.

\section{Author details}

${ }^{1}$ Department of Hygiene and Public Health, School of Medicine, Teikyo University, 2-11-1 Kaga, Itabashi-ku, Tokyo 173-8605, Japan. ${ }^{2}$ Department of Health and Social Behavior, School of Public Health, The University of Tokyo, 7-3-1 Hongo, Bunkyo-ku, Tokyo 113-0033, Japan. ${ }^{3}$ Department of International and Community Oral Health, Tohoku University Graduate School of Dentistry, 4-1 Seiryo-machi, Aoba-ku, Sendai, Miyagi 980-8575, Japan.

Received: 30 April 2014 Accepted: 15 September 2014

Published: 19 September 2014

\section{References}

1. Murata C, Yamada T, Chen CC, Ojima T, Hirai H, Kondo K: Barriers to health care among the elderly in Japan. Int J Environ Res Public Health 2010, 7:1330-1341.

2. Grytten J, Holst D, Skau I: Demand for and utilization of dental services according to household income in the adult population in Norway. Community Dent Oral Epidemiol 2012, 40:297-305.

3. Lu JF, Leung GM, Kwon S, Tin KY, van Doorslaer E, O'Donnell O: Horizontal equity in health care utilization evidence from three high-income Asian economies. Soc Sci Med 2007, 64:199-212.

4. van Doorslaer E, Masseria C: Income-related inequality in the use of medical care in 21 OECD countries. In Towards High-Performing Health Systems: Policy Studies. Edited by Organisation for Economic Co-operation and Development. Paris: OECD Publishing; 2004:109-165.

5. Maharani DA, Rahardjo A: Is the utilisation of dental care based on need or socioeconomic status? A study of dental care in Indonesia from 1999 to 2009. Int Dent J 2012, 62:90-94.

6. Somkotra $T$, Detsomboonrat $P$ : Is there equity in oral healthcare utilization: experience after achieving Universal Coverage. Community Dent Oral Epidemiol 2009, 37:85-96.

7. Haugejorden O, Klock KS, Astrom AN, Skaret E, Trovik TA: Socio-economic inequality in the self-reported number of natural teeth among Norwegian adults - an analytical study. Community Dent Oral Epidemiol 2008, 36:269-278.

8. Wamala S, Merlo J, Bostrom G: Inequity in access to dental care services explains current socioeconomic disparities in oral health: the Swedish National Surveys of Public Health 2004-2005. J Epidemiol Community Health 2006, 60:1027-1033.

9. Hjern A, Grindefjord M, Sundberg H, Rosen M: Social inequality in oral health and use of dental care in Sweden. Community Dent Oral Epidemiol 2001, 29:167-174.

10. Ikegami N, Yoo BK, Hashimoto H, Matsumoto M, Ogata H, Babazono A, Watanabe R, Shibuya K, Yang BM, Reich MR, Kobayashi Y: Japanese universal health coverage: evolution, achievements, and challenges. Lancet 2011, 378:1106-1115.

11. Shika hoken kenkyukai: Zenka jitsurei ni yoru shakai hoken shika shinryo, 2013. Tokyo: 2013. in Japanese. 
12. Sintonen $\mathrm{H}$, Linnosmaa I: Economics of dental services. In Handbook of Health Economics, Volume 1B. Edited by Culyer AJ, Newhouse JP. Amsterdam: Elsevier; 2000:1251-1296.

13. Ministry of Health, Labour and Welfare: The National Health and Nutrition Survey in Japan, 2009. Tokyo: 2012. in Japanese.

14. Moriya S, Ando $\mathrm{Y}$, Miura H: Trends and prospects of oral health conditions among Japanese: the achievement of 8020. J Natl Inst Public Health 2011, 60:379-386. in Japanese, only abstract in English.

15. Kiyak HA, Reichmuth M: Barriers to and enablers of older adults' use of dental services. J Dent Educ 2005, 69:975-986.

16. Lorenc $T$, Petticrew $M$, Welch $V$, Tugwell P: What types of interventions generate inequalities? Evidence from systematic reviews. J Epidemiol Community Health 2013, 67:190-193.

17. Takada M, Kondo N, Hashimoto H, for the J-SHINE Data Management Committee: Japanese Study on Stratification, Health, Income, and Neighborhood: study protocol and profiles of participants. J Epidemiol 2014, 24:334-344.

18. Hagenaars AJM, de Vos K, Zaidi MA: Poverty statistics in the late 1980s: research based on micro-data. Luxembourg: Office for Official Publications of the European Communities; 1994

19. Jones JA, Spiro A 3rd, Miller DR, Garcia Rl, Kressin NR: Need for dental care in older veterans: assessment of patient-based measures. J Am Geriatr Soc 2002, 50:163-168.

20. Jones JA, Kressin NR, Miller DR, Orner MB, Garcia RI, Spiro A 3rd: Comparison of patient-based oral health outcome measures. Qual Life Res 2004, 13:975-985.

21. Hanibuchi T, Aida J, Nakade M, Hirai H, Kondo K: Geographical accessibility to dental care in the Japanese elderly. Community Dent Health 2011 , 28:128-135.

22. Grignon $M$, Hurley J, Wang $L$, Allin S: Inequity in a market-based health system: evidence from Canada's dental sector. Health Policy 2010, 98:81-90.

23. Listl S: Income-related inequalities in dental service utilization by Europeans aged 50+. J Dent Res 2011, 90:717-723.

24. Krieger N, Chen JT, Selby JV: Comparing individual-based and householdbased measures of social class to assess class inequalities in women's health: a methodological study of 684 US women. J Epidemiol Community Health 1999, 53:612-623.

25. Honjo K, Iso H, Iwata M, Cable N, Inoue M, Sawada N, Tsugane S, JPHC Study Group: Effectiveness of the combined approach for assessing social gradients in stroke risk among married women in Japan. J Epidemiol 2012, 22:324-330.

26. Sakurai K, Kawakami N, Yamaoka K, Ishikawa H, Hashimoto H: The impact of subjective and objective social status on psychological distress among men and women in Japan. Soc Sci Med 2010, 70:1832-1839.

27. Green CA, Pope CR: Gender, psychosocial factors and the use of medical services: a longitudinal analysis. Soc Sci Med 1999, 48:1363-1372.

28. Nguyen L, Hakkinen U: Income-related inequality in the use of dental services in Finland. Appl Health Econ Health Policy 2004, 3:251-262.

29. Moeller JF, Chen H, Manski RJ: Investing in preventive dental care for the Medicare population: a preliminary analysis. Am J Public Health 2010, 100:2262-2269.

30. Lowe C, Blinkhorn AS, Worthington HV, Craven R: Testing the effect of including oral health in general health checks for elderly patients in medical practice - a randomized controlled trial. Community Dent Oral Epidemiol 2007, 35:12-17.

31. Sato M: Present and future of economic evaluation in the field of dentistry. $J$ Health Care Soc 2002, 12:117-134. in Japanese, only abstract in English.

32. Tolonen H, Dobson A, Kulathinal S, WHO MONICA Project: Effect on trend estimates of the difference between survey respondents and non-respondents: results from 27 populations in the WHO MONICA Project. Eur J Epidemiol 2005, 20:887-898.

33. Laaksonen M, Aittomäki A, Lallukka T, Rahkonen O, Saastamoinen P, Silventoinen K, Lahelma E: Register-based study among employees showed small nonparticipation bias in health surveys and check-ups. J Clin Epidemiol 2008, 61:900-906.

34. Toyokawa S, Kobayashi Y: Increasing supply of dentists induces their geographic diffusion in contrast with physicians in Japan. Soc Sci Med 2010, 71:2014-2019.
35. Galobardes B, Shaw M, Lawlor DA, Lynch JW, Davey SG: Indicators of socioeconomic position (part 1). J Epidemiol Community Health 2006, $60: 7-12$.

36. Ministry of Health, Labour and Welfare: The Comprehensive Survey of the Living Conditions of People on Health and Welfare in Japan, 2010. Tokyo: 2012 (in Japanese).

doi:10.1186/1472-6831-14-117

Cite this article as: Murakami et al:: Income-related inequalities in preventive and curative dental care use among working-age Japanese adults in urban areas: a cross-sectional study. BMC Oral Health 2014 14:117.

\section{Submit your next manuscript to BioMed Central and take full advantage of:}

- Convenient online submission

- Thorough peer review

- No space constraints or color figure charges

- Immediate publication on acceptance

- Inclusion in PubMed, CAS, Scopus and Google Scholar

- Research which is freely available for redistribution 